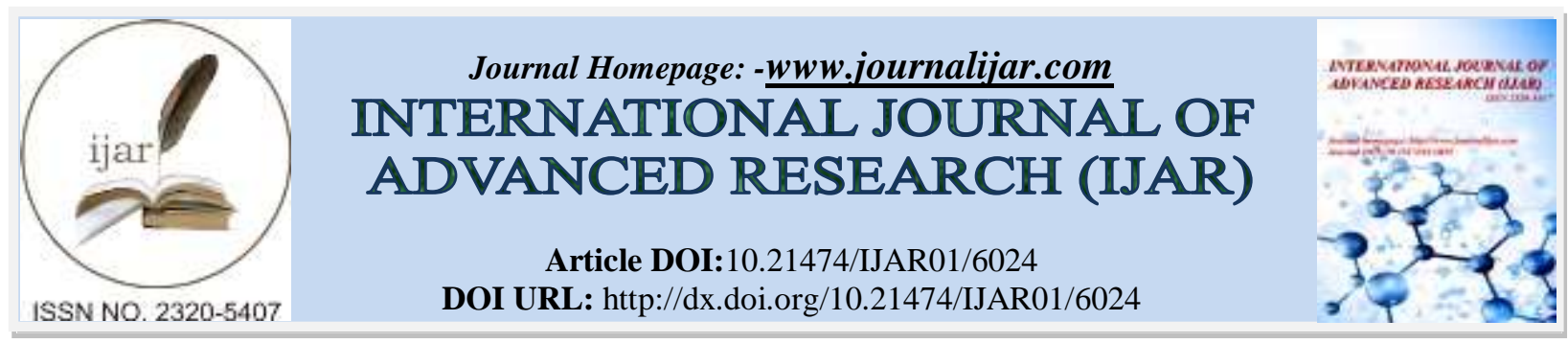

RESEARCH ARTICLE

\title{
A REVIEW ARTICLE ON THE FORMATION, MECHANISM AND BIOCHEMISTRY OF MDA AND MDA AS A BIOMARKER OF OXIDATIVE STRESS.
}

Saima Jadoon and Arif Malik.

Institute of Molecular Biology and Biotechnology, University of Lahore, Pakistan.

\section{Manuscript Info}

Manuscript History

Received: 10 October 2017

Final Accepted: 12 November 2017

Published: December 2017

Key words:-

Biomarker, carbon compounds, oxidative stress, reactive oxygen species.

\begin{abstract}
Malondialdehyde (MDA) as a biomarker is widely used for examining oxidative stress in biomedical field where Lipid peroxidation is a chain phenomenon resulting in the formation of various active compounds that result in cellular damage. Bio monitoring of MDA is used as a biomarker in different diseases like in-vivo and in vitro. MDA is also used in various types of diseases like hypertension, diabetes, heart failure and cancer etc. Higher level of MDA was found in patients that suffer from various types of diseases including lung cancer, complex original pain syndrome. In finding the oxidative stress MDA is used as a reliable biomarker. MDA is found in high levels in patients that suffer with goiter. MDA determination can take place by different methods. The determination of MDA was studied in goiter patients through a simple, and rapid but sensitive scientific method. This review is aimed to study the biochemical mechanism of formation of maliondialdehyde and the role of Maliondialdehyde as a biomarker for the diagnosis of several biochemicalcompetition.
\end{abstract}

Copy Right, IJAR, 2017,. All rights reserved.

\section{Introduction:-}

Malondialdehyde which is an organic substance is a by-product of polyunsaturated fatty acids $\left(\mathrm{C}_{7} \mathrm{H}_{16} \mathrm{O}_{4}\right)$. This naturally existing specie is reactive and is a marker of oxidative stress and is used for the diagnosis of many diseases. Malondialdehyde is the most reactive dialdehyde. It is composed of three carbons which are produced as a result of different process like by-product of polyunsaturated fatty acids peroxidation and arachidonic acid metabolism. Malondialdehyde is the by-product of these metabolisms (Fruhbeck et al., 2001, Frayan et al., 1998). Malondialdehyde is the most reactive compound, so as a result it readily combines with the different functional groups on the molecules like proteins, lipoproteins and DNA. Due to its high reactivity it reacts with these functional groups very easily and it does not need any external force like heat or temperature etc. The proteins which are MDA-proteins may show different chemical behaviors. Malondialdehyde is the organic compound which also contains some particular formula $\mathrm{CH}_{2}(\mathrm{CHO})_{2}$. The formula seems to be simple but its structural formula is very complex than that of its molecular formula. In laboratory, the hydrolysis of 1, 1, 3, 3-tetramethoxypropane results in the synthesis of MDA (Vance and J.E Vance., 2002).

\section{Biomarker of Lipid Peroxidation:-}

The process of stealing electrons by free radicals from lipids is known as lipid per oxidation which harms cells significantly. In general this process affects polyunsaturated fatty acids because they contain multiple bonds that possess reactive hydrogen. Lipid peroxides are the integral part of polyunsaturated fatty acids, whose decomposition 
results into variety of carbonyl compounds. The most widespread is maliondialdehyde (Massey and Nicolauo., 2011, Massy and Nicolauo., 2013). MDA is a compound, the target of the reactive specie is the C-C double bond of poly-unsaturated fatty acids which weakens $\mathrm{C}-\mathrm{H}$ bond, a free radical is formed which can obstruct the hydrogen atom and a liquid free radical is formed which suffers oxidation generating peroxyl radical. This peroxyl radical reacts with other polyunsaturated fatty acids. This process can be propagated continually in a chain reaction (Jornayvaz and Shulman, 2012). During this process the formation of lipidhydro peroxide is unstable and its fragmentation yields products such as maliondialdehyde. Lipid peroxidation is well-established mechanism, of cellular energy in both plants and animals. Therefore, the presence of lipid peroxidation is used as a measure of MDA. Enol is the most common form MDA is usually found in both plants and animals. However, a cis-trans system is present in organic solvents. But it shows that the maliondialdehyde is present in both forms (Yang and Kaznietz., 2013). Malondialdehyde is commonly observed in the form of 1, 1, 3, 3 tetramethoxypropaneWhen this compound is hydrolyzed then maliondialdehyde is produced. In the synthesis of thromboxane A2, MDA is a byproduct. In cyclogenase 1 or cyclogenase 2 metabolizes arachnoidic acid to prostaglandin $\mathrm{H} 2$ by platelets the product is further metabolized to thromboxane A2, 12 hydroxyhepatadecatrinoic acid and MDA (Banumann et al., 2013).

\section{MDA, Lipid Peroxidation and Mutation In Hummain Cells:-}

MDA is the indicator of lipid per oxidation. During the process of lipid per oxidation many different products are formed. Among these products, MDA is also formed which is mutagenic (Fisher et al., 2002). MDA plays a vital role in damaging DNA and endogenously mutation occurs in cells. MDA modifies the replication process of single stand DNA and causes mutation of Guanine $\rightarrow$ Thymine, Adenine $\rightarrow$ Guanine and Cytosine $\rightarrow$ Thymine (Conway \& Miller, 2007, Takuwa et al., 2012).

\section{Biochemistry Of Malondialdehyde:-}

Reactive oxygen species degrade the polyunsaturated lipids. The maliondialdehyde are prepared by the polyunsaturated lipids. Inside DNA, they react with deoxyadenosine and deoxyguanosine. Malondialdehyde measures oxidative stress in human body. In human body maliondialdehyde plays an important role (Maltson., 2003 ) in which aldehyde is one of the most reactive electrophile. The amount of tissues is helpful in eliminating the degree of lipid peroxidation in maliondialdehyde. The maliondialdehyde plays very important role in human body. DNA in which deoxyaelenosine is the derivative of nucleoside adenosine. It accumulates T-Lymphocytes in the human body. It kills those cells resulting in a genetic disorder which is called as adenosine deminase (Hannun and Obeid et al., 2008). The other is deoxyguanosine. The deoxyguanosine is an adduct of MDA and DNA bases. Malondialdehyde measures the oxidative stress in human body. Any disturbance between the reactive oxygen species and the ability of the biological to remove the harmful substances and repair the resulting damage is known as oxidative stress (Aoki and Narumiya., 2012,Tang et al.,2012, ). The spread of aspergs syndrome in human body depends upon oxidative stress. Oxidative metabolism causes base damage in the DNA. The reactive oxygen species are the main factor that induce and cause serious damage in DNA. On other hand, reactive oxygen species play very important role in immune system in attacking and killing pathogens, and hinders the aging process (Kalinski., 2012). The maliondialdehyde is prepared by polyunsaturated lipids. The polyunsaturated lipids are hydrocarbon chains having two or more $\mathrm{C}=\mathrm{C}$ bond. Nuts, seeds and fish etc are most common source of polyunsaturated fats.

The unsaturated fats refer the fact that the molecules have fewer quantity of hydrogen. Malondialdehyde and other thiobarbituric acid reactive substances give fluorescence. The thiobarbituric acid is an oxygenic compound and the hetroacyclic. It is used as a reagent in assaying maliondialdehyde. It is mostly used in the lipid peroxidation. It is also used as a biomarker in the maliondialdehyde (Kay and Grinstein et al., 2013).

Maliondialdehyde is the end product of lipid peroxidation. As said earlier, MDA is reactive in nature and has the potential for causing mutation. Edibles like sunflower and palm oil contain MDA in good quantity. The reactive oxygen species in MDA cause serious damage to cell structures. Certain known reasons play key role in increasing the degree of reactive oxygen species. These are the demise species containing oxygen. The examples of reactive oxygen species are presidepuperenide, hydroxyl radicals and single oxygen (Pluchino et al., 2013). Maliondialdehydedialdehyde are common in organic chemistry. Many fragrances are aldehydes. Under isolated conditions, MDA can be directly measured by using HPLC. MDA which is an indicator of cell membrane injury is the most common residue produced during the process of lipid per oxidation. Peroxidation, oxidative stress can be measured and evaluated by means of the level of maliondialdehyde in different tissues (Moldovan and N.I Moldovan ., 2004). 
In maliondialdehyde, the concentrations of adenine nucleotide derivatives were also obtained in the same chromatographic run. Under the experimental conditions no detachable amount of maliondialdehyde was observed (Halliwell and Gutteridge., 1984). MDA formed as a result of the breakdown of poly unsaturated lipids by reactive exygen species forming maliondialdehyde represents an aldehyde family as electrophile specie and has the ability to cause toxic stress in cells and form covalent protein adduct.

Inside DNA, MDA reacts with deoxyadenosine to form a mutagenic DNA adduct known as MG which is an indicator of oxidative stress in organism (Venero et al., 2003).

\section{Increased Level of Lipid Peroxidation Assosiated With Variety of Diseases:-}

Malondialdehyde in combination with other thiobarbituric reactive substances (TBARS) form bio substances that are the root cause of several chronical and other diseases in humans (Castellani et al., 2004). Many diseases of liver are the result of oxidative stress. Also there exist a relationship of oxidative stress indicator among hepatic tissue, hepatic and peripheral veins and urine. Aging, neurodegerative diseases, chronic inflammatory disease and several types of cancers are caused by oxidative stress. Biomedical research has shown that MDA is also found in tissues, and sections of joints in patients suffering from osteoarthritis (Lipinski and Pretorius., 2012). Some other diseases caused by oxidative stress include (but not limited to) Parkinson's disease, Alzheimer's disease, atherosclerosis, heart failure, myocardial infarction etc. (Bielski et al., 1983).

\section{Malondialdehyde (MDA) As A Diagnostic Biomarker:-}

Malondialdehyde is most commonly used as an indicator in some experiments like lipid peroxidation (Dizdaroglu and Jaruga et al., 2012). It is a chain of reactions which takes place during the oxidative stress including propanediol and 4-hyrdoxyrnonenal (HNE) resulting in the cellular damage. Issues related with the validity of biomarker have hindered the process of lipid peroxidation. The plasma concentration of maliondialdehyde (P-MDA) is frequently used biomarker for the study of lipid peroxidation. (Kanno et al., 2012). It is one of several by-products of lipid peroxidation process. Smoking is one of the risk factors for increased lipid peroxidation; it is due to the presence of free radical in cigarette smoke. The level of plasma maliondialdehyde (P-MDA) also increases in cigarette smokers. reactive oxygen species produced in human body are protected by the antioxidant sytem present in the body. Superoxide radicals after being converted into hydrogen peroxide $\left(\mathrm{H}_{2} \mathrm{O}_{2}\right)$ are released from the body with the help of superoxide dismutases (SOD). In the presence of catalyze (enzyme) hydrogen peroxide is converted into water and oxygen. Similar function is performed by glutathione peroxidases in the removal of $\mathrm{H}_{2} \mathrm{O}_{2}$ from the body.

Malondialdehyde is a major lipid peroxidation process and acts as an indicator in the assessment of cancer risk in human beings. In order to detect DNA damage in human oral mucosa a special monoclonal antibody which is specific for MDA, DNA adduct has been developed and is also useful for the endogenous agent in oxidative stress and carcinogenesis. MDA as an endogenous product participates in various biochemical reaction i-e., covalent bonds in protein. Cyclic adducts are generated when MDA reacts with deoxygenases. The major DNA adduct is a pyrimidopurinone of deoxyguanosine. In bacterial and mammalian cells MDA is mutagenic and can cause cancer in rats (Schneider et al., 2008). MDA is still used as a biomarker of oxidative stress in clinical investigation. MDA is the biomarker of oxidative stress in many health problem such as cancer, psychiatry, chronic obstructive pulmonary disease and asthma or cardiovascular problems. Thiobarbituric acid the most commonly used method for the determination of MDA in biological fluid. This assay based on the condensation reaction of TBA and MDA in which reaction rate depend on temperature, $\mathrm{pH}$ and concentration. The reaction takes place in acidic solution. Most of MDA is produced during reaction process from decomposition of products. The rapidity ease of use and cost of TBA made it the most common method (Browne and Armstrong., 2000). Non specificity of TBA reactivity on MDA and production of MDA from reactivity of MDA from other than lipid peroxidation effect of procedural modification on MDA-TBA reactive substances act as a biomarker of oxidative stress instead of MDA values of oxidative stress instead of MDA value. Effect of procedural modification on MDA -TBA adducts development. Low stability of MDA in biological samples is due to the reason of its high tendency for reaching with protein, amino acid (Yin et al., 2011).

\section{Malondialdehyde As Diagnosis of Diabetes Mellitus:-}

Malondialdehyde is the by-product of the lipid oxidation which is present in the free radical. Malondialdehyde is the very toxic by product which is derived from the lipid oxidation. The studies has revealed that its high concentration is present in the diabetes mellitus. The patients that are suffered from the diabetes mellitus have the high quantity of 
the maliondialdehyde. Malondialdehyde react irreversible and reversible with the proteins and phosphorus to obtain the good result (Volinsky and Kinnunen., 2013).

\section{Malondialdihyde is Mutagenic In Human Cell:-}

Malondialdehyde is a genotoxic product of different activities like enzymatic activities or oxygen radical-induced lipid peroxidation. The analysis of the sequences revealed that MDA include some mutations that take place at base pairs. The most common mutations that happened are insertions and deletions. MDA is completely abolished when the adducted shuttle vector was replicated lacking nucleotide excision repair (Kinnunen et al., 2012).

\section{Relation Of Lipid Perxidation And DNA Oxidation:-}

Lipid peroxidation is the free radical mediated which is in the form of chain reactions. These reactions initiated a number of toxic products when they are allowed to propagate in the biological membranes. MDA is produced by different mechanisms and it is a three carbon product with les molecular weight. In MDA the species mainly target at the $\mathrm{C}=\mathrm{C}$ bond of the polyunsaturated fatty acids. This $\mathrm{C}=\mathrm{C}$ bond affect the carbon-hydrogen bond and weaken them and due to this the hydrogen is easily abstracted. This lipid peroxidation is unstable and it form different products like maliondialdehyde. For monitoring MDA level in different biological systems, lipid peroxidation serves as a vital tool both in-vitro (outside a living organism) and in-vivo (taking place in a living organism) for various health disorders (Reis and Spickett., 2012).

\section{Malondialdehyde And Heavy Metals:-}

Plants contain different types of microelements among these copper is considered an essential microelements. These micro elements are required for biological system such as structural and catalytical component of proteins and enzymes. Copper and zinc super oxide are also used and are involved in the process of electron transport chain in photosynthesis. The excess of heavy metals is harmful for plants as excess of heavy metal in cells cause molecular damage to plants in both ways either directly or indirectly. Along side these heavy metals, some protective enzymatic mechanisms and non-enzymatic mechanism are present. The antioxidant enzymes are superoxide dismutase, peroxides and catalase. Garlic (allium sativum) is reported because it has the ability to tolerate the heavy stresses including but not limited to heavy metal stress (Yin and Xu., 2011). 
Fig.1:- Formation of MDA (Patra et al., 2011).

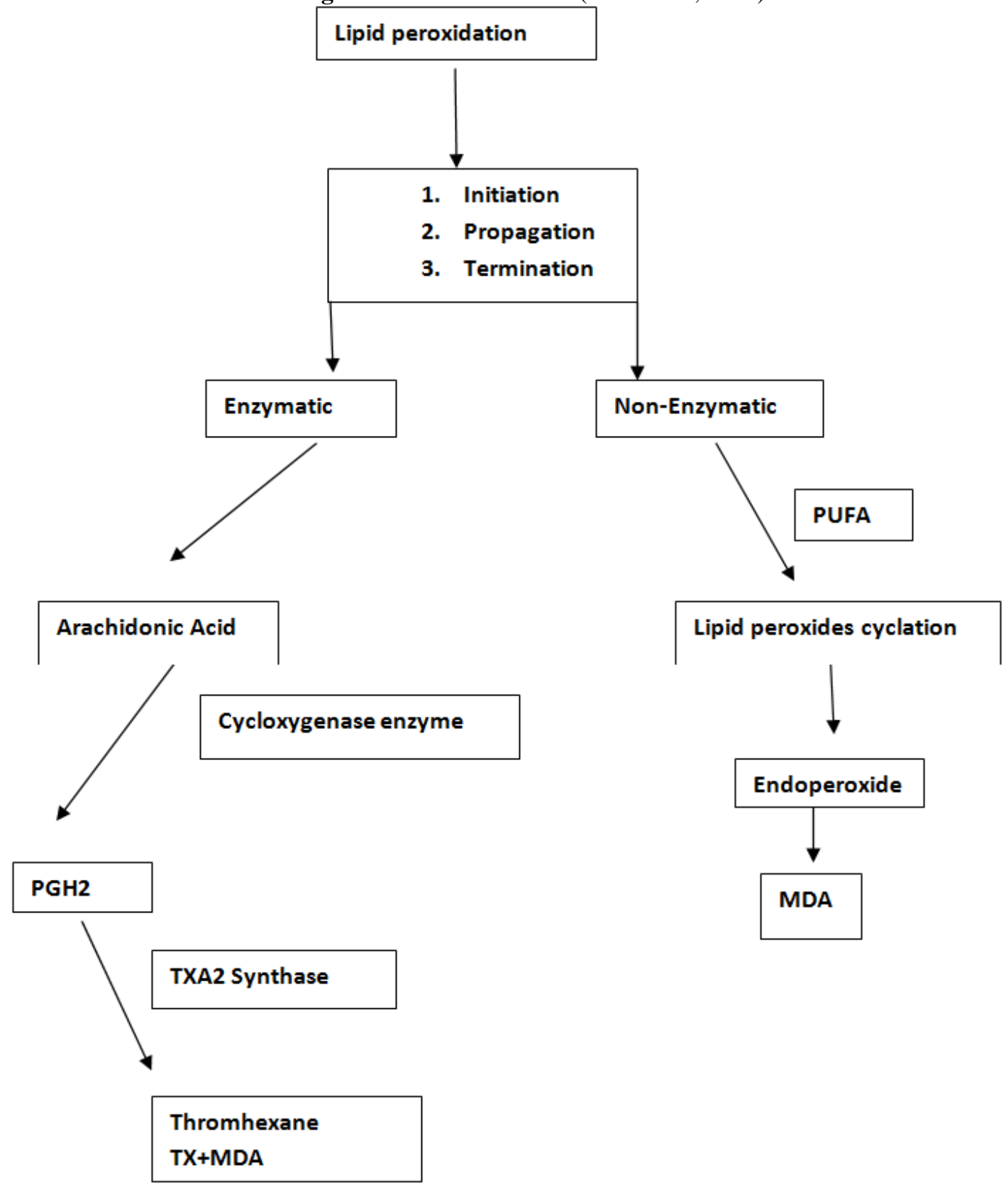


Fig.2:- Formation of MDA on cell membrane of human beings due to heavy metals (Ayala et al., 2014, Giorg et al., 2010).

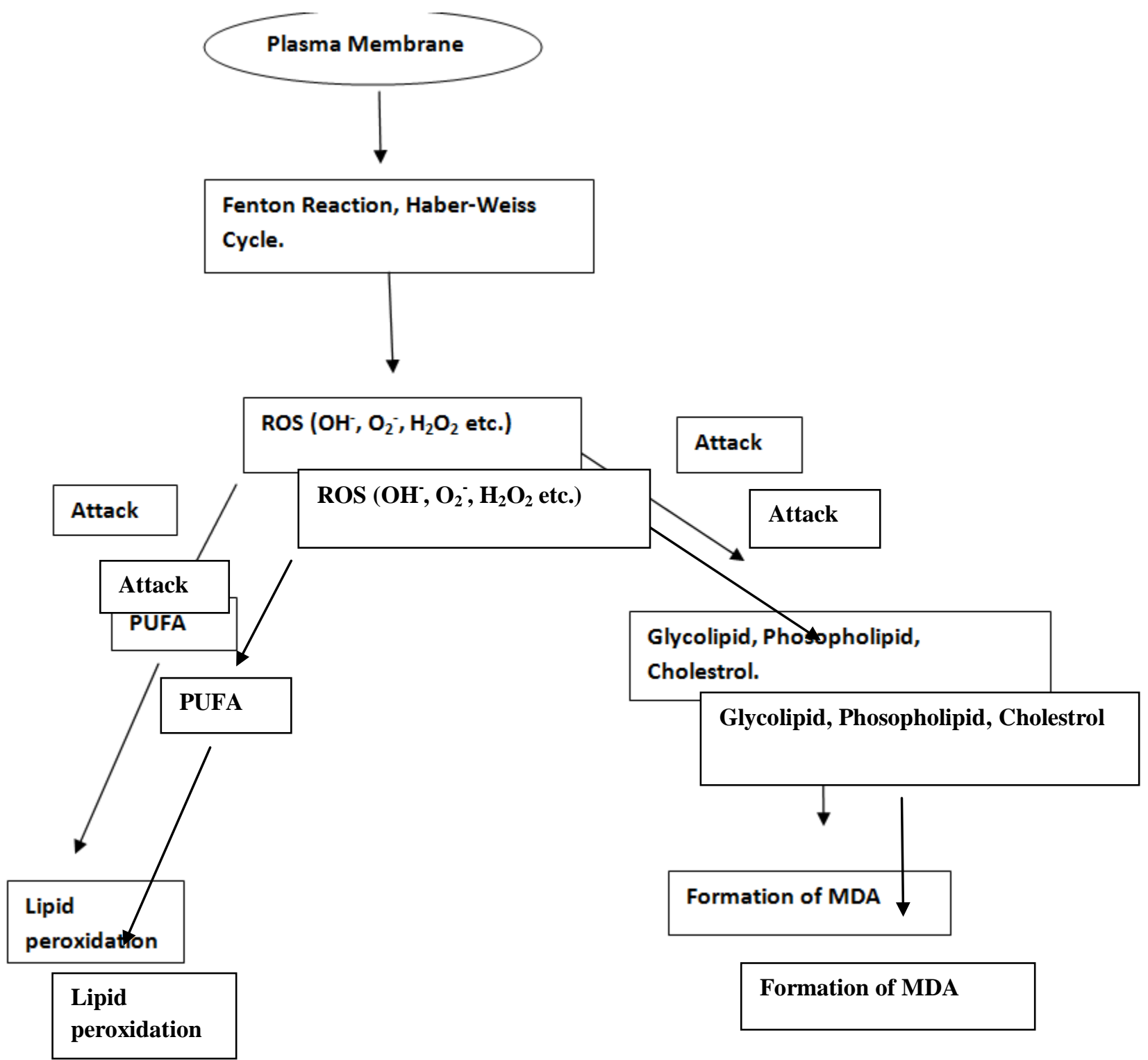

Explanation of Fig.1, 2:-ROS are formed due to Fenton reaction and Haber weiss cycle. Polyunsaturated fatty acids (PUFA) inside the plasma membrane. Most strongly ROS attack on PUFA and lipid peroxidation happens. The enzymes lipoxygenase, cycloxygenase, cytochroms P 450. It also causes membrane impairment. PUFA breaks double bond and forms lipidperoxide and hydrolipid peroxide. It further attacks on Glycolipids, Phosopholipids and cholesterol which are present as a secondary product and it forms MDA (Patra et al., 2011). MDA forms in two ways one is enzymatic and second is non enzymatic metabolism. In enzymatic MDA the arachidonic acid is present in our body and converts into the prostaglandin- $\mathrm{H}_{2}$ in the presence of cyclooxygenase enzyme. We can say that prostaglandin $-\mathrm{H}_{2}$ is indication of disease and then $\mathrm{PGSH}_{2}$ convert into thermobohexane and MDA in the presence of thermbohexane synthase. Oxidation of MDA occurs with the help of mitochondrial aldehyde as a result it produce acetalaldehyde then through aldehyde dehydrogenaes enzyme acetyl CoA and covert in water and carbon dioxide. In case of non-enzymatic when ROS attack on PUFA and process of lipid peroxidation occurs. Lipid peroxides and through cyclosation process changes in to bicyclic endoperoxide and further changes in to MDA without any enzyme and further changes in to MDA without involvement of any enzyme. It reacts with protein and DNA in cell 
and then form adduct of MDA and cause cellular damage.MDA acts as a signaling messenger, regulate the genetic expression, cause the mutation and breaks DNA strand. It also arrests the cell cycle and induces the apoptosis (Ayala et al., 2014,Giorg et al., 2010).

\section{Conclusion:-}

MDA is a byproduct of polyunsaturated fatty acids. It is the biomarker of lipid peroxidation. Heavy metals act as a ligand attached with receptor of plasma membrane and produce ROS. PUFA bonds break due to lipid peroxidation. MDA act as signaling messenger. It regulates genetic expression, cause mutation and break DNA strand. MDA acts as a signaling messenger regulating the gene expression, and increased level of MDA promot islets GSIS and elevate ATP , cytosolic $\mathrm{Ca}^{+2}$ and affect on protein activity.

\section{References:-}

1. Aoki.T and S.Narumiya (2012).Prostaglandins and chronic inflammation. Trends in Pharmacological Sciences, 33(6): 304-311.

2. Ayala. A., M.F Muno, and D.Swarup (2011). Oxidative stress in lead and cadmium toxicity and it amelioration. Vet Med int 2011(2011).

3. Bielski B.H.J., R. L. Arudi and M. W. Sutherland (1983).A study of the reactivity of $\mathrm{HO}_{2} / \mathrm{O}_{2}$ - with unsaturated fatty acids. Journal of Biological Chemistry, 258(8):4759-4761.

4. Baumann J, C. Sevinsky, and D. S. Conklin (2013).Lipid biology of breast cancer. BiochimicaetBiophysicaActa, 1831(10): 1509-1517.

5. Browne R.Wand D. Armstrong (2000). HPLC analysis of lipidderived polyunsaturated fatty acid peroxidation products in oxidatively modified human plasma. Clinical Chemistry, 46(6):829-836.

6. Castellani R.J, K. Honda, X. Zhu et al (2004). "Contribution of redox-active iron and copper to oxidative damage in Alzheimer disease.Ageing Research Reviews, 3(3): 319-326.

7. Conway S.J and G. J. Miller (2007).Biology-enabling inositol phosphates, phosphatidylinositol phosphates and derivatives. Natural Product Reports, 24(4): 687-707.

8. Dizdaroglu M and P. Jaruga (2012). Mechanisms of free radicalinduced damage to DNA.Free Radical Research, 46:382-419.

9. Fruhbeck G., J. G omez-Ambrosi, F. J. Muruzabal, and M. A. Burrell (2001).The adipocyte: a model for integration of endocrine and metabolic signaling in energy metabolism regulation. The American Journal of Physiology: Endocrinology and Metabolism, vol. 280(6): 827-847.

10. Frayn.K.N., (1998). Regulation of fatty acid delivery in vivo. Advances in Experimental Medicine and Biology, 441: 171-179.

11. Fisher S.K, J. E. Novak, and B.W. Agranoff (2002).Inositol and higher inositol phosphates in neural tissues: homeostasis, metabolism and functional significance. Journal of Neurochemistry, 82(4):736-754.

12. Fruhwirth.G.O, A. Loidl, and A. Hermetter (2007).Oxidized phospholipids: from molecular properties to disease. BiochimicaetBiophysicaActa: Molecular Basis of Disease, 1772(7): 718-736.

13. Giorgi.C, C. Agnoletto, C. Baldini et al (2010).Redox control of protein kinase C: cell-and disease-specific aspects. Antioxidants and Redox Signaling, 13 (7), 1051-1085.

14. Hannun Y.A and L. M. Obeid (2008). "Principles of bioactive lipid signalling: lessons from sphingolipids.Nature Reviews Molecular Cell Biology, 9(2):139-150.

15. Halli.Bwell and J. M. C. Gutteridge, "Oxygen toxicity, oxygen radicals, transition metals and disease," Biochemical Journal, 219(1):1-14.

16. Jornayvaz F.R and G. I. Shulman (2012).Diacylglycerol activation of protein kinase C $\varepsilon$ and hepatic insulin resistance. Cell Metabolism, 15(5): 574-584.

17. Kalinski.P (2012). Regulation of immune responses by prostaglandin E2. Journal of Immunology, 188 (1): 2128.

18. Kay J.G., and S. Grinstein (2013). Phosphatidylserine-mediated cellular signaling. Advances in Experimental Medicine and Biology, 99: 177-193.

19. Kanno T., K. Nakamura, H. Ikai, K. Kikuchi, K. Sasaki, and Y. Niwano (2012). Literature review of the role of hydroxyl radicals in chemically-induced mutagenicity and carcinogenicity for the risk assessment of a disinfection system utilizing photolysis of hydrogen peroxide.Journal of Clinical Biochemistry and Nutrition, vol. 51(1): 9-14. 
20. Kinnunen P.K.J., K. Kaarniranta, and A. K. Mahalka (2012). Proteinoxidized phospholipid interactions in cellular signaling for cell death: from biophysics to clinical correlations.BiochimicaetBiophysicaActa, 1818(10): 2446-2455.

21. Lipinski B and E. Pretorius (2012).Hydroxyl radical-modified fibrinogen as a marker of thrombosis: the role of iron. Hematology, 17(4):241-247.

22. Lane N (2002). Oxygen: The Molecule that Made the World, Oxford University Press.

23. Massey K.A., and A. Nicolaou (2011). Lipidomics of polyunsaturatedfatty-acid-derived oxygenated metabolites. Biochemical Society Transactions, vol. 39(5)1240-1246.

24. Massey K.A and A. Nicolaou (2013).Lipidomics of oxidized polyunsaturated fatty acids. Free Radical Biology and Medicine, 59: 45-55 .

25. Mattson M.P (2003). Membrane Lipid Signaling in Aging and AgeRelated Disease. Elsevier , 12: 263.

26. Moldovan L and N. I. Moldovan (2004).Oxygen free radicals and redox biology of organelles. Histochemistry and Cell Biology, 122(4) : 395-412.

27. Pluchino .N., M. Russo, A. N. Santoro, P. Litta, V. Cela, and A. R. Genazzani(2013).Steroid hormones and BDNF. Neuroscience, 239: 271-279.

28. Reis A and C. M. Spickett (2012).Chemistry of phospholipid oxidation. BiochimicaetBiophysicaActa, 1818(10):2374- 2387.

29. Schneider C., W. E. Boeglin, H. Yin, N. A. Porter, and A. R. Brash (2008). Intermolecular peroxyl radical reactions during autoxidation of hydroxy and hydroperoxyarachidonic acids generate a novel series of epoxidized products. Chemical Research in Toxicology, 21(4): 895-903.

30. Takuwa Y, Y. Okamoto, K. Yoshioka, and N. Takuwa, (2012).Sphingosine-1-phosphate signaling in physiology and diseases. Bio Factors, 38 (5): 329-337.

31. Tang E.H.C, P. Libby, P. M. Vanhoutte, and A. Xu (2012).Antiinflammation therapy by activation of prostaglandin EP4 receptor in cardiovascular and other inflammatory diseases. Journal of Cardiovascular Pharmacology, 59(2): 116-123.

32. Vance E and J. E. Vance (2002). Biochemistry: Biochemistry of Lipids. Lipoproteins and Membranes, 4th edition.

33. Venero J.L, M. Revuelta, L. Atiki et al (2003) .Evidence for dopamine derived hydroxyl radical formation in the nigrostriatal system in response to axotomy. Free Radical Biology and Medicine, 34(1):111-123.

34. Yin $\mathrm{H}, \mathrm{L}$. $\mathrm{Xu}$, and N. A. Porter (2011).Free radical lipid peroxidation: mechanisms and analysis. Chemical Reviews, 111(10): 5944-5972.

35. Volinsky R and P. K. J. Kinnunen (2013). Oxidized phosphatedylcholines in membrane-level cellular signaling: from biophysics to physiology and molecular pathology. FEBS Journal, 280(12):2806-2816.

36. Yang C and M. G. Kazanietz (2007). Chimaerins: GAPs that bridge diacyl glycerol signalling and the small Gprotein Rac. Biochemical Journal, 403(1):1-12. 